\title{
Identification of resistant germplasm containing novel resistance genes at or tightly linked to the Pi2/9 locus conferring broad-spectrum resistance against rice blast
}

Gui Xiao ${ }^{1,2}$, Frances Nikki Borja', Ramil Mauleon'1, Jonas Padilla', Mary Jeanie Telebanco-Yanoria', Jianxia Yang ${ }^{3}$, Guodong Lü ${ }^{3}$, Maribel Dionisio-Sese ${ }^{2}$ and Bo Zhou ${ }^{{ }^{*}}$ (I)

\begin{abstract}
Background: The rice Pi2/9 locus harbors multiple resistance $(R)$ genes each controlling broad-spectrum resistance against diverse isolates of Magnaporthe oryzae, a fungal pathogen causing devastating blast disease to rice. Identification of more resistance germplasm containing novel $R$ genes at or tightly linked to the Pi2/9 locus would promote breeding of resistance rice cultivars.

Results: In this study, we aim to identify resistant germplasm containing novel $R$ genes at or tightly linked to the Pi2/9 locus using a molecular marker, designated as Pi2/9-RH (Pi2/9 resistant haplotype), developed from the $5^{\prime}$ portion of the Pi2 sequence which was conserved only in the rice lines containing functional Pi2/9 alleles. DNA analysis using Pi2/9-RH identified 24 positive lines in 55 shortlisted landraces which showed resistance to 4 rice blast isolates. Analysis of partial sequences of the full-length cDNAs of Pi2/9 homologues resulted in the clustering of these 24 lines into 5 haplotypes each containing different Pi2/9 homologues which were designated as Pi2/9-A5, -A15, -A42, -A53, and -A54. Interestingly, Pi2/9-A5 and Pi2/9-A54 are identical to Piz-t and Pi2, respectively. To validate the association of other three novel Pi2/9 homologues with the blast resistance, monogenic lines at $\mathrm{BC}_{3} \mathrm{~F}_{3}$ generation were generated by marker assisted backcrossing (MABC). Resistance assessment of the derived monogenic lines in both the greenhouse and the field hotspot indicated that they all controlled broad-spectrum resistance against rice blast. Moreover, genetic analysis revealed that the blast resistance of these three monogenic lines was co-segregated with Pi2/9-RH, suggesting that the Pi2/9 locus or tightly linked loci could be responsible for the resistance.

Conclusion: The newly developed marker Pi2/9-RH could be used as a potentially diagnostic marker for the quick identification of resistant donors containing functional Pi2/9 alleles or unknown linked $R$ genes. The three new monogenic lines containing the Pi2/9 introgression segment could be used as valuable materials for disease assessment and resistance donors in breeding program.
\end{abstract}

Keywords: Magnaporthe oryzae, Pi2/9 homologues, Resistant haplotype specific marker

\footnotetext{
* Correspondence: b.zhou@irri.org

${ }^{1}$ Genetics and Biotechnology Division, International Rice Research Institute,

DAPO Box 7777, Metro Manila, Philippines

Full list of author information is available at the end of the article
} 


\section{Background}

Rice blast, a devastating rice disease caused by the fungal pathogen Magnaporthe oryzae, is affecting rice production across all rice-growing areas worldwide (Ashkani et al. 2014). Introgression of resistance $(R)$ genes into rice cultivars remains the most economical and effective approach for rice blast disease management (Ashkani et al. 2015; Tanweer et al. 2015). The bottleneck of this approach is that, after an individual $R$ gene is isolated and deployed in the varieties, it can be overcome in a short time (usually in 2-3 years) by the emergence of a compatible pathogen because of the high level of avirulence (Avr) effector variability in the pathogen (Skamnioti and Gurr 2009; Valent and Khang, 2010). Therefore, it is essential to enrich and diversify the $R$-gene pool by extensive and continuous exploration of novel $R$ genes or alleles in diverse germplasm for the choice of effective $R$ genes in a rice resistance breeding program.

To date, more than 100 rice blast $R$ genes and over 350 resistance quantitative trait loci (QTLs) have been genetically identified (Tanweer et al. 2015). Of the $100 R$ genes, 25 were molecularly characterized (Liu et al. 2013; Fukuoka et al. 2014; Su et al. 2015; Ma et al. 2015; Chen et al. 2015). Most of them encode proteins having nucleotide binding site (NBS) and leucine-rich repeat (LRR) domains. It is evident that many NBS-LRR-type $R$ genes are organized as alleles located at the same loci (Leung et al. 2015). For example, at least eight Pik alleles were molecularly characterized at the Pik locus, which is located on the distal end of the long arm of chromosome 11 (Chen et al. 2015; Campbell et al. 2004; Ashikawa et al. 2008; Yuan et al. 2011; Zhai et al. 2011; Hua et al. 2012; Ashikawa et al. 2012; Zhai et al. 2014). It is worth noting that many $R$-gene alleles are extremely sequence-related to each other. For example, Pik-1, one of two NBS-LRR genes at the Pik locus in Kusabue, differs from its allele Pik1-KA in Kanto51 by only four nucleotides confined in the region encoding the NBS domain (Zhai et al. 2011; Ashikawa et al. 2012). Other NBS-LRR genes, Pik-2 and Pik2-KA, are even identical to each other (Zhai et al. 2011; Ashikawa et al. 2012). A similar scenario was also observed at the Pish locus (Takahashi et al. 2010).

Several approaches were employed for the identification of novel $R$ genes or alleles of known $R$ loci, such as map-based cloning, allele mining and genome-wide association study (GWAS). Recently, 97 loci associated with rice blast resistance were identified using the GWAS approach (Kang et al. 2015). By combining the RNA interference (RNAi) approach, the candidate gene in LABR_64 corresponding to resistance to all five isolates was validated and confirmed to be an allele of Pi5. In addition to the traditional gene-linked markers (Wang et al. 1994; Fjellstrom et al. 2006; Hayashi et al. 2004; Thakur et al. 2014), gene-specific or diagnostic markers were recently reported for the identification of novel blast $R$ genes or alleles in diverse germplasm, such as Pi54 (Ramkumar et al. 2011), Pikm (Costanzo and Jia, 2010) and Pike (Chen et al. 2015).

The Pi2/9 locus located on the short arm of chromosome 6 proximal to the centromere was reported to harbor at least eight functional alleles from different donor varieties (Su et al. 2015; Qu et al. 2006; Zhou et al. 2006; Deng et al. 2006; Jeung et al. 2007; Wang et al. 2012; Jiang et al. 2012). Analyses of genetic diversity of the Pi2/9 homologues in cultivar and wild rice species revealed that the Pi2/9 homologues were subjected to strong diversifying selection (Zhou et al. 2007; Dai et al. 2010; Liu et al. 2011). Molecular characterization of Pi2, Pi9, Piz- $t$ and $P i 50$ revealed that a limited number of sequence variations disproportionately confined within the LRR regions of the encoded $R$ proteins mainly determined the distinct recognition specificities of these alleles to different sets of rice blast isolates ( $\mathrm{Su}$ et al. 2015; Zhou et al. 2006; Qu et al. 2006). Moreover, these alleles are each embedded within a cluster containing multiple sequence-related paralogues at the locus in the respective donor varieties (Su et al. 2015; Zhou et al. 2006; Qu et al. 2006). The feature of the complex organization of highly sequencerelated homologues at the Pi2/9 locus makes it difficult to develop gene-specific molecular markers for the diagnosis and identification of known and novel alleles from diverse germplasm. Intriguingly, contrasting to the absence in susceptible rice varieties, the $\mathrm{Pi2}$ alleles are exclusively present in the resistant haplotypes (Su et al. 2015; Zhou et al. 2007), prompting us an assumption that the Pi2 sequence could be targeted for developing markers for allele mining at the Pi2/9 locus. In this study, we aim to develop a resistant haplotype specific marker at the Pi2/9 locus and apply it for the identification of novel resistant germplasm containing new Pi2/9 alleles. The development of introgression lines via marker-assisted backcrossing $(\mathrm{MABC})$ enabling the analysis of resistance spectra of these novel introgression lines against a wide collection of rice blast isolates in the Philippines will be also described.

\section{Results \\ Development and validation of the Pi2/9 resistant haplotype marker, $\mathrm{Pi} 2 / 9-\mathrm{RH}$}

The availability of medium-depth coverage of genome sequences of 3024 rice accessions allowed us to search the existence of the alleles of Nbs2-Pi2 and Nbs4-Pi2 (Pi2) in a large rice collection (The 3000 Rice Genomes Project, 2014). It was found that only 12 out of 3024 lines had very limited or even no sequences reads aligning to the promoter of $\mathrm{Nbs} 2-\mathrm{Pi} 2$ (corresponding to the region of chromosome 6 at the position: 10,380,244$10,381,506 \mathrm{bp}$ in pseudomolecule 1.0 of MSU Rice Genome Annotation Project Release 7-RGAP 7.0, http:// 
rice.plantbiology.msu.edu), suggesting that almost all the sequenced accessions contain the alleles of Nbs2-Pi2 (Fig. 1). On the contrary, only 691 out of 3024 lines were found to have sequence reads matching the promoter of $\mathrm{Pi} 2$ [corresponding to the region of the Pi2 locus (Genbank accession no. DQ352453) at the position: $72,301-73,620 \mathrm{bp}$ ], indicating that about $23 \%$ of the rice accessions contain the alleles of Pi2 (Fig. 1). The disproportionate distribution pattern of Nbs2-Pi2 and Pi2 alleles in $3 \mathrm{~K}$ genomes prompted us to further investigate whether the existence of the Pi2-unique sequence can infer to the existence of functional Pi2/9 alleles in the diverse germplasm. A unique sequence fragment corresponding to the promoter and a portion of the first intron of Pi2 were selected as the region for the development of a resistant haplotype specific marker so called Pi2/9-RH for the PCR screening. Two pairs of primers (Pi2/9-DF1/DR1 and Pi2/9-DF2/DR2) were synthesized and optimized with excellent PCR amplification efficiency (Table 1). Because of the close locations of these two primer pairs, only Pi2/9-DF1/DR1 was used as Pi2/9$\mathrm{RH}$ in this study.

We firstly used LTH-derived International Rice Research Institute (IRRI) bred blast resistant lines (IRBLs) containing different $R$ genes for the analysis. Four IRBLs containing Pi2, Pi9, Piz-t and Piz were each resolved with a PCR amplicon at the expected size using Pi2/9$\mathrm{RH}$. On the contrary, no PCR amplification was resolved in other IRBLs containing non-Pi2/9 genes and five susceptible rice varieties (Additional file 1: Figure S1). We further tested the existence of $\mathrm{Pi} 2 / 9-\mathrm{RH}$ in five rice panels. In the panel of 50 accessions showing susceptibility to at least one of two isolates (Panel IV) from the rice $2 \mathrm{~K}$ panel (http://ricephenonetwork.irri.org/diversity-panels), only $6 \%$ of 50 rice lines showed positive PCR amplification by Pi2/9-RH (Table 2). It was also

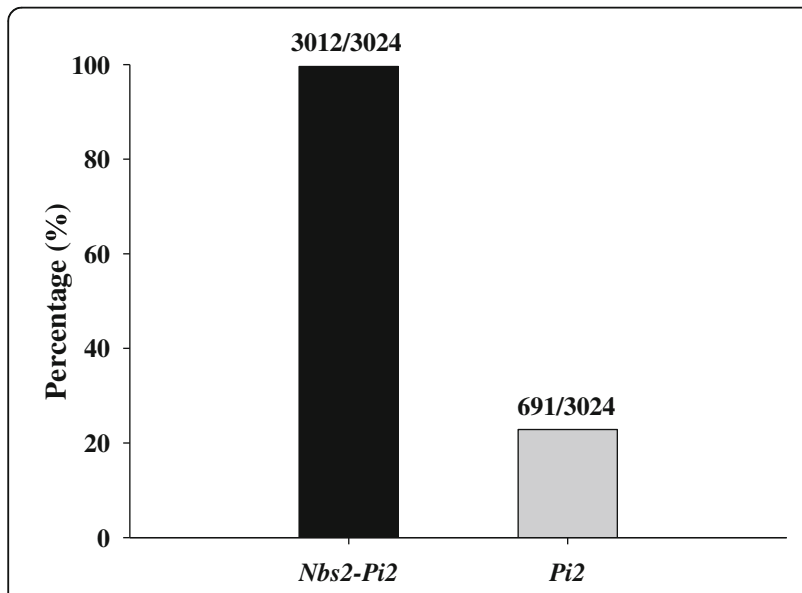

Fig. 1 Frequency of Nbs2-Pi2 and Pi2 alleles in 3024 rice germplasm accessions. The figure was constructed using the program of Sigmaplot found that only $8 \%$ of 50 IRRI released varieties and advanced breeding lines (Panel V) showed positive PCR amplification. On the contrary, the resistant rice lines obtained by screening against different number of rice blast isolates showed $24.4 \%$ and $45.5 \%$ frequency of positive PCR amplification, respectively, in Panel III and I (Table 2). Moreover, the rice lines resistant to more isolates displayed a higher frequency than the ones resistant to fewer isolates (45.5\% in Panel I versus $24.4 \%$ in Panel III) (Table 2 and Additional file 2: Table S1). These data suggested a positive correlation between the frequency of the existence of $\mathrm{Pi} 2 / 9-\mathrm{RH}$ and resistance to rice blast in the rice panels.

\section{Identification of five Pi2/9 haplotype groups each containing different Pi2/9 homologues in resistant rice germplasm}

The identification of $26 \mathrm{Pi} / 9-\mathrm{RH}$ positive lines in Panel I (Additional file 2: Table S1) promoted us to investigate whether they contain known or novel Pi2/9 homologues except A16 which was resolved with a PCR amplicon of a larger size (Additional file 1: Figure S2). We attempted the cloning of full-length coding sequences (CDSs) using conserved primers 5UTR-F/3UTR-R (Table 1) and proceeded for sequencing. Cluster analysis based on the partial sequence of the CDSs resulted in five unique haplotypes each containing different $P i 2 / 9$ homologues which were named $P i 2 / 9-A 5,-A 15,-A 42,-A 53$, and $-A 54$ (Table 3$)$. Interestingly, $P i 2 / 9-A 5$ and $-A 54$ were identical in sequence to Piz-t and Pi2, respectively, which was further confirmed by sequencing the entire CDS (Table 3$)$. On the contrary, Pi2/9-A15, $-A 42$ and - $A 53$ each contained sequence differences from any of the known Pi2/9 alleles, suggesting these haplotypes contained novel Pi2/9 homologues.

It was found that these 25 rice lines originated from different countries in Asia, Africa, Europe and South America, representing a wide geographic distribution in the world (Table 3). Some Pi2/9 alleles were identified in multiple rice lines from different regions, for example, Pi2/9-A42 was present in 14 rice lines. Moreover, 12 out of 14 rice lines belonged to the subgroup of tropical japonica, representing a disproportional distribution of Pi2/9-A42 in different rice subgroups (Table 3).

\section{Introgression lines containing 3 novel Pi2/9 haplotypes showed broad-spectrum resistance against rice blast isolates in the Philippines}

To validate the association between the Pi2/9 haplotypes and resistance against rice blast, we generated $\mathrm{BC}_{3} \mathrm{~F}_{3}$ introgression lines of Pi2/9-A15, Pi2/9-A42 and Pi2/9$A 53$ in the background of the susceptible variety CO39 via MABC using the marker of Pi2/9-RH and named the derived monogenic lines as IR126181, IR126183 and 
Table 1 Primers used in this study

\begin{tabular}{llll}
\hline Primer name & Primer sequence $\left(5^{\prime}\right.$ to $\left.3^{\prime}\right)$ & Purpose & Expected Size $(\mathrm{bp})$ \\
\hline Pi2/9-DF1 & CTTGACATCCAAACCGCACC & For the development of the marker Pi2/9-RH \\
Pi2/9-DR1 & TAGGCCTAGCCAATTTTGCC & \\
Pi2/9-DF2 & CAGACGCTGCCGAAGGCTGC & For the development of the marker Pi2/9-RH \\
Pi2/9-DR2 & CAATAGTTGCTGATTCCTGAGC & \\
5UTR-F & CTTGAAGGGAGAGTCGAACG & For the cloning of full-length cDNA of Pi2/9 homologues \\
3UTR-R & GCCTCATTGATCATCATGCC & & 1512 \\
\hline
\end{tabular}

IR126184, respectively. These 3 introgression lines together with IRBLs were assessed to a set of 34 CO39-virulent isolates from a diverse collection in the Philippines for the resistance spectrum analysis. As Table 4 indicated, both IR126181 (Pi2/9-A15) and IR126184 (Pi2/9-A53) were resistant to all isolates as observed in IRBL9-W (Pi9). IR126183 (Pi2/9-A42) was resistant to 24 isolates and susceptible to 4 isolates. Intriguingly, IR126183 showed partial resistance to four isolates that developed typical type- 3 lesions on the leaves (Table 4 and Additional file 1: Figure S3). Out of these 34 isolates, 2 and 23 were virulent to IRBL-z5[CO] (Pi2) and IRBL-zt[CO] (Piz-t), respectively (Table 4).

To evaluate the resistance of these three introgression lines in the field, we tested them with different IRBLs in a field hot spot in Bohol, the Philippines in 2016. They all showed strong resistance whereas LTH and CO39 showed high susceptibility to blast in the field (Fig. 2). Similar to these 3 introgression lines, IRBLz5-CA (Pi2) and IRBL9-W showed strong resistance. On the contrary, IRBLzt-T (Piz- $t$ ) was susceptible. Taken together, we postulated that these 3 introgression lines IR126181, IR126183 and IR126184 controlled broad-spectrum resistance against rice blast isolates in the Philippines (Fig. 2).

\section{The resistance of introgression lines was associated with the Pi2/9 haplotypes}

To validate whether the resistance of the introgression lines was associated with the Pi2/9 haplotypes, each

Table 2 Frequency of Pi2 orthologues in different rice panels diagnosed by using the primer pair $\mathrm{Pi} 2 / 9-\mathrm{RH}$

\begin{tabular}{llll}
\hline Rice panel & $\begin{array}{l}\text { Number of accessions } \\
\text { assessed }\end{array}$ & $\begin{array}{l}\text { Number of positive } \\
\text { accessions }\end{array}$ & Frequency (\%) \\
\hline $\mathrm{I}^{\mathrm{b}}$ & 55 & 25 & 45.5 \\
$\mathrm{II}^{\mathrm{c}}$ & 156 & 38 & 24.4 \\
$\mathrm{IV}^{\mathrm{d}}$ & 50 & 4 & 8.0 \\
$\mathrm{~V}^{\mathrm{e}}$ & 50 & 3 & 6.0 \\
\hline
\end{tabular}

${ }^{a}$ The information of rice accessions and reactions to different rice blast isolates

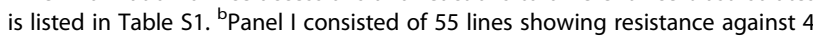
isolates. A16 was not counted in the frequency calculation due to the amplicon with an unexpected size. 'Panel III consisted of 156 lines showing resistance against two rice blast isolates. ${ }^{\mathrm{d}}$ Panel IV consisted of 50 randomly selected lines from the IRRI rice $2 \mathrm{~K}$ diversity panel. ${ }^{e}$ Panel $\mathrm{V}$ consisted of 50 IRRI-released varieties and advanced lines
$\mathrm{BC}_{3} \mathrm{~F}_{2}$ population used for the advancement of $\mathrm{BC}_{3} \mathrm{~F}_{3}$ introgression lines was proceeded with genetic analysis. As Table 5 listed, 550 out of 720 progenies of the $\mathrm{BC}_{3} \mathrm{~F}_{2}$ population of Pi2/9-A15 were resistant whereas 170 progenies were susceptible to the isolate MO15-21, displaying an expected 3:1 ratio of resistance versus susceptibility. The same ratio was also observed for resistant versus susceptible progenies of the $\mathrm{BC}_{3} \mathrm{~F}_{2}$ population of Pi2/9-A53 against the isolate $5167-1$. These data indicated that the resistance of Pi2/9-A15 and Pi2/9-A53 introgression lines was each controlled by a single genetic locus. For the case of Pi2/9-A42, an expected 15:1 ratio of resistance versus susceptibility was observed in the $463 \mathrm{BC}_{3} \mathrm{~F}_{2}$ progenies to the isolate MO15-21, indicating that the resistance was controlled by two individual genetic loci. To further validate the linkage of resistance to the Pi2/9 locus, all the susceptible plants for each $\mathrm{BC}_{3} \mathrm{~F}_{2}$ population underwent genotyping with $\mathrm{Pi} 2 / 9-\mathrm{RH}$. None of the susceptible progenies produced the positive PCR amplicon (Table 5), indicating that the resistance could be tightly linked with the marker of Pi2/9-RH. We thus speculated that the Pi2/9 locus or tightly linked one was likely responsible for the resistance observed in the introgression lines of Pi2-A15, Pi2-A42 and Pi2-A53.

\section{Discussion}

An integrated approach combines germplasm screening by resistant haplotype specific marker, sequencing of full-length cDNA and development of monogenic lines for efficient identification of resistant germplasm containing putatively functional Pi2/9 alleles

Exploration of novel blast $R$ genes or alleles is a continuous effort to ensure the selection of the most effective ones for breeding resistant rice varieties. In addition to the traditional map-based cloning strategy, several approaches were successfully developed for the fast characterization of $R$ genes, such as GWAS (Kang et al. 2015), MutMap-Gap (Takagi et al. 2013) and $R$-gene analog (RGA)-based linkage analysis coupled with mutant characterization (Okuyama et al. 2011). Thanks to the advances in sequencing technology and increasing genome information, allele mining provides a powerful and economical approach for the identification of novel alleles (Bhullar et al. 2010). For example, nine new alleles of Pi54 containing sequence differences from known ones were identified 
Table 3 Clustering of 25 Pi2/9-RH positive rice lines by sequencing the Pi2/9 homologues

\begin{tabular}{|c|c|c|c|c|c|}
\hline $\begin{array}{l}\text { Pi2/9 } \\
\text { homologues }\end{array}$ & $\begin{array}{l}\text { Number of accessions carrying } \\
\text { the same homologues }\end{array}$ & $\begin{array}{l}\text { IRGC accession } \\
\text { number }\end{array}$ & $\begin{array}{l}\text { Varietal } \\
\text { group }\end{array}$ & $\begin{array}{l}\text { Country of } \\
\text { origin }\end{array}$ & Remark \\
\hline \multirow[t]{5}{*}{ Pi2/9-A5 } & \multirow[t]{5}{*}{5} & 121299 & Indica & Thailand & \multirow{24}{*}{$\begin{array}{l}\text { Identical to Piz- } t \text { in nucleotide } \\
\text { sequence }\end{array}$} \\
\hline & & 121383 & Indica & Lao PDR & \\
\hline & & 121392 & Indica & Bangladesh & \\
\hline & & 121753 & Indica & Philippines & \\
\hline & & 121898 & Aus/boro & India & \\
\hline \multirow[t]{4}{*}{ Pi2/9-A15 } & \multirow[t]{4}{*}{4} & 121434 & Indica & China & \\
\hline & & 121607 & Indica & India & \\
\hline & & 121611 & Indica & Chinese Taiwan & \\
\hline & & 121660 & Indica & Philippines & \\
\hline \multirow[t]{14}{*}{ Pi2/9-A42 } & \multirow[t]{14}{*}{14} & 121315 & TropJap & Colombia & \\
\hline & & 121490 & TropJap & Bolivia & \\
\hline & & 121536 & TropJap & Côte d'Ivoire & \\
\hline & & 121632 & Indica & Colombia & \\
\hline & & 121698 & TropJap & Philippines & \\
\hline & & 121699 & TropJap & Madagascar & \\
\hline & & 121730 & TropJap & Madagascar & \\
\hline & & 121744 & TropJap & France & \\
\hline & & 121749 & TropJap & Philippines & \\
\hline & & 121755 & TropJap & Philippines & \\
\hline & & 121762 & TropJap & Ghana & \\
\hline & & 121764 & TropJap & Côte d'Ivoire & \\
\hline & & 121804 & Indica & Colombia & \\
\hline & & 121805 & TropJap & Colombia & \\
\hline Pi2/9-A53 & 1 & 121884 & Indica & Vietnam & \\
\hline Pi2/9-A54 & 1 & 121888 & Indica & Panama & Identical to Pi2 in nucleotide sequence \\
\hline
\end{tabular}

IRGC International Rice Genebank Collection, TropJap tropical japonica

in 885 Indian rice genotypes via allele mining (Vasudevan et al. 2015). Functional validation of alleles containing genetic variations followed by identification is another key step in allele mining, which is usually carried out by gene complementation tests, silencing or knockout approaches. For example, the function of Pid3-A4, a novel allele of Pid3 identified in the common wild rice A4 by referring to the sequence of Pid 3 in cultivated rice, was validated by generation and resistance assessment of Pid3-A4 transgenic plants against rice blast isolates ( $\mathrm{Lv}$ et al. 2013). However, the fact that rice blast $R$ genes are often organized in complexes and are highly similar in sequence from one another hinders the efficient identification and functional validation of candidate genes in the process of allele mining. In this regard, development of diagnostic markers becomes a prerequisite for efficient screening of known alleles of $R$ genes in diverse germplasm. The Pi2/9 locus is one of the complex loci which were extensively investigated for the identification of different alleles in diverse germplasm by using various gene-linked or gene-specific markers including simple sequence repeat (SSR), insertion/deletion (InDel), and cleavage amplified polymorphisms (CAPS) markers (Jiang et al. 2015; Liu et al. 2002; Hayashi et al. 2006; Zhu et al. 2012; Tian et al. 2016). These markers were used either for the identification of particular alleles in contained populations or for the diagnosis of known alleles in different germplasm. For the latter case, however, it is really necessary to validate the correlation between the existence of know alleles and the polymorphic pattern of markers, particularly for gene linked SSR markers, due to the extreme sequence similarity among different functional and nonfunctional alleles at the Pi2/9 locus (Su et al. 2015; Zhou et al. 2007). Moreover, those markers have limited application in the identification of novel alleles at the Pi2/9 locus. It is indeed that, similarly to the $P i 2 / 9$ gene family, most rice blast $R$ genes are classified into Type-II $R$ gene family whose members differ by a limited number of point mutations (Luo et al. 2012). Thus, it is valuable to develop an approach 
Table 4 Disease reaction patterns of the introgression lines IR126181 (Pi2/9-A15), IR126183 (Pi2/9-A42), IR126184 (Pi2/9-A53), IRBL-z5[CO] (Pi2), IRBL-zt[CO] (Piz-t) and IRBL-9W (Pi9) and two susceptible lines, CO39 and Lijiangxintuanheigu (LTH), against 34 M. oryzae isolates ${ }^{a}$

\begin{tabular}{|c|c|c|c|c|c|c|c|c|}
\hline \multirow[t]{2}{*}{ Isolate } & \multirow[t]{2}{*}{ CO39 } & \multicolumn{3}{|c|}{ New introgression lines ${ }^{b}$} & \multicolumn{3}{|c|}{ Pi2/9 known allele introgression lines ${ }^{c}$} & \multirow[t]{2}{*}{ LTH } \\
\hline & & $\begin{array}{l}\text { IR126181 } \\
\text { (Pi2/9-A15) }\end{array}$ & $\begin{array}{l}\text { IR126183 } \\
\text { (Pi2/9-A42) }\end{array}$ & $\begin{array}{l}\text { IR126184 } \\
\text { (Pi2/9-A53) }\end{array}$ & IRBL-z5[CO] (Pi2) & $\begin{array}{l}\text { IRBL-zt[CO] } \\
(\text { Piz-t) }\end{array}$ & IRBL-9 W (Pi9) & \\
\hline BN111 & $\mathrm{S}$ & $\mathrm{R}$ & $\mathrm{R}$ & $\mathrm{R}$ & $\mathrm{R}$ & $\mathrm{S}$ & $\mathrm{R}$ & S \\
\hline BN209 & $S$ & $\mathrm{R}$ & NA & $\mathrm{R}$ & S & S & $\mathrm{R}$ & S \\
\hline CA41 & $\mathrm{S}$ & $\mathrm{R}$ & R & $\mathrm{R}$ & R & $\mathrm{R}$ & R & S \\
\hline CA89 & $S$ & $\mathrm{R}$ & $P R$ & $\mathrm{R}$ & $\mathrm{R}$ & S & $\mathrm{R}$ & S \\
\hline IK81-25 & $\mathrm{S}$ & $\mathrm{R}$ & R & $\mathrm{R}$ & R & S & R & S \\
\hline JMB8401 & $S$ & $\mathrm{R}$ & R & $\mathrm{R}$ & $\mathrm{R}$ & S & $\mathrm{R}$ & S \\
\hline JMB840610 & $S$ & $\mathrm{R}$ & S & $\mathrm{R}$ & $\mathrm{R}$ & $\mathrm{R}$ & $\mathrm{R}$ & S \\
\hline M36-1-3-10-1 & $\mathrm{S}$ & $\mathrm{R}$ & R & $\mathrm{R}$ & R & $\mathrm{R}$ & R & S \\
\hline M39-1-3-8-1 & S & $\mathrm{R}$ & R & $\mathrm{R}$ & R & $\mathrm{R}$ & R & S \\
\hline M64-1-3-9-1 & $\mathrm{S}$ & $\mathrm{R}$ & S & $\mathrm{R}$ & $\mathrm{R}$ & $\mathrm{R}$ & $\mathrm{R}$ & S \\
\hline PO6-6 & $\mathrm{S}$ & $\mathrm{R}$ & R & $\mathrm{R}$ & R & S & R & S \\
\hline V86010 & S & $\mathrm{R}$ & S & $\mathrm{R}$ & $\mathrm{R}$ & $\mathrm{R}$ & $\mathrm{R}$ & S \\
\hline 5008-3 & $\mathrm{S}$ & $\mathrm{R}$ & R & $\mathrm{R}$ & $\mathrm{R}$ & S & R & S \\
\hline $5092-3$ & $\mathrm{~S}$ & $\mathrm{R}$ & R & $\mathrm{R}$ & $\mathrm{R}$ & S & $\mathrm{R}$ & S \\
\hline $5167-1$ & $\mathrm{~S}$ & $\mathrm{R}$ & NA & $\mathrm{R}$ & S & $\mathrm{R}$ & R & S \\
\hline 6161-1 & $S$ & $\mathrm{R}$ & R & $\mathrm{R}$ & $\mathrm{R}$ & S & $\mathrm{R}$ & S \\
\hline $9126-1$ & $\mathrm{~S}$ & $\mathrm{R}$ & R & $\mathrm{R}$ & R & S & R & S \\
\hline $9244-3$ & $S$ & $\mathrm{R}$ & R & $\mathrm{R}$ & R & S & $\mathrm{R}$ & S \\
\hline $9406-3$ & $\mathrm{~S}$ & $\mathrm{R}$ & R & $\mathrm{R}$ & R & S & R & S \\
\hline $9475-1-3$ & $\mathrm{~S}$ & $\mathrm{R}$ & $\mathrm{R}$ & $\mathrm{R}$ & R & S & R & S \\
\hline $9482-1-3$ & $S$ & $\mathrm{R}$ & S & $\mathrm{R}$ & R & R & R & S \\
\hline $9497-3$ & $S$ & $\mathrm{R}$ & $P R$ & $\mathrm{R}$ & R & S & R & S \\
\hline IBN008 & $S$ & $\mathrm{R}$ & R & $\mathrm{R}$ & R & S & R & S \\
\hline IBN028 & $\mathrm{S}$ & $\mathrm{R}$ & R & $\mathrm{R}$ & R & S & R & S \\
\hline MO15-1 & $S$ & $\mathrm{R}$ & R & $\mathrm{R}$ & R & S & R & S \\
\hline MO15-6 & $\mathrm{S}$ & $\mathrm{R}$ & PR & $\mathrm{R}$ & R & S & R & S \\
\hline M015-19 & S & $\mathrm{R}$ & R & $\mathrm{R}$ & R & R & R & S \\
\hline MO15-21 & $\mathrm{S}$ & $\mathrm{R}$ & R & $\mathrm{R}$ & R & $S$ & R & S \\
\hline M015-24 & $\mathrm{S}$ & $\mathrm{R}$ & R & $\mathrm{R}$ & R & R & R & S \\
\hline M015-27 & $\mathrm{S}$ & $\mathrm{R}$ & PR & $\mathrm{R}$ & R & S & R & S \\
\hline MO15-110 & S & $\mathrm{R}$ & R & $\mathrm{R}$ & R & S & R & S \\
\hline MO15-125 & $\mathrm{S}$ & $\mathrm{R}$ & R & $\mathrm{R}$ & R & R & R & S \\
\hline MO15-226 & $\mathrm{S}$ & $\mathrm{R}$ & R & $\mathrm{R}$ & R & $S$ & R & $S$ \\
\hline MO15-244 & $S$ & $\mathrm{R}$ & R & $\mathrm{R}$ & R & S & R & S \\
\hline
\end{tabular}

${ }^{a} \mathrm{R}$ indicates resistance, $\mathrm{PR}$ indicates partial resistance, $\mathrm{S}$ indicates susceptibility and NA indicates not available. Resistance evaluations are based on the $0-5$ scale of the Standard Evaluation System. ${ }^{\mathrm{b}}$ The three new introgression lines are all in the CO39 genetic background. ${ }^{\mathrm{C}} \mathrm{IRBL}-\mathrm{z} 5[\mathrm{CO}]$ and IRBL-zt[CO] are introgression lines of Pi2 and Piz- $t$ in the genetic background of CO39; IRBL9-W is the introgression line of the Pi9 gene in the genetic background of LTH

to quickly identify germplasm containing novel Pi2/9 alleles before the extensive molecular characterization. In this study, we developed an integrated approach for the efficient identification of germplasm showing broad-spectrum resistance against blast resistance which was likely conferred by novel Pi2/9 alleles or their tightly linked genetic loci. First, the resistant haplotype specific marker Pi2/9-RH was developed and used for the identification of germplasm containing putative Pi2/9 alleles for further functional characterization. Different from other allele- 
A

\begin{tabular}{clccccc}
\hline \multirow{2}{*}{ Label } & \multirow{2}{*}{ Material } & \multicolumn{3}{c}{ Score (per replicate) } & \multirow{2}{*}{ Mean } & \multirow{2}{*}{ Phenotype } \\
\cline { 3 - 5 } & & 1 & 2 & 3 & & \\
\hline a & CO39 & 9 & 9 & 9 & 9.0 & $\mathrm{~S}$ \\
$\mathrm{~b}$ & IR126181 (Pi2/9-A15) & 4 & 2 & 3 & 3.0 & $\mathrm{R}$ \\
$\mathrm{c}$ & IR126183(Pi2/9-A42) & 3 & 1 & 3 & 2.3 & $\mathrm{R}$ \\
$\mathrm{d}$ & IR126184 (Pi2/9-A53) & 3 & 2 & 3 & 2.7 & $\mathrm{R}$ \\
$\mathrm{e}$ & Lijiangxintuanheigu (LTH) & 9 & 9 & 9 & 9.0 & $\mathrm{~S}$ \\
$\mathrm{f}$ & IRBLz5-CA $($ Pi2) & 2 & 1 & 3 & 2.0 & $\mathrm{R}$ \\
$\mathrm{g}$ & IRBL9-W $($ Pi9 $)$ & 3 & 1 & 1 & 1.7 & $\mathrm{R}$ \\
$\mathrm{h}$ & IRBLzt-T $($ Piz- $t)$ & 9 & 8 & 9 & 8.7 & $\mathrm{~S}$ \\
\hline
\end{tabular}

For 0-9 scale: score 0-3 indicates resistant (R), 4-5 indicates moderately resistant (MR), 6

B

indicates moderately susceptible (MS) and 7-9 indicates susceptible (S).

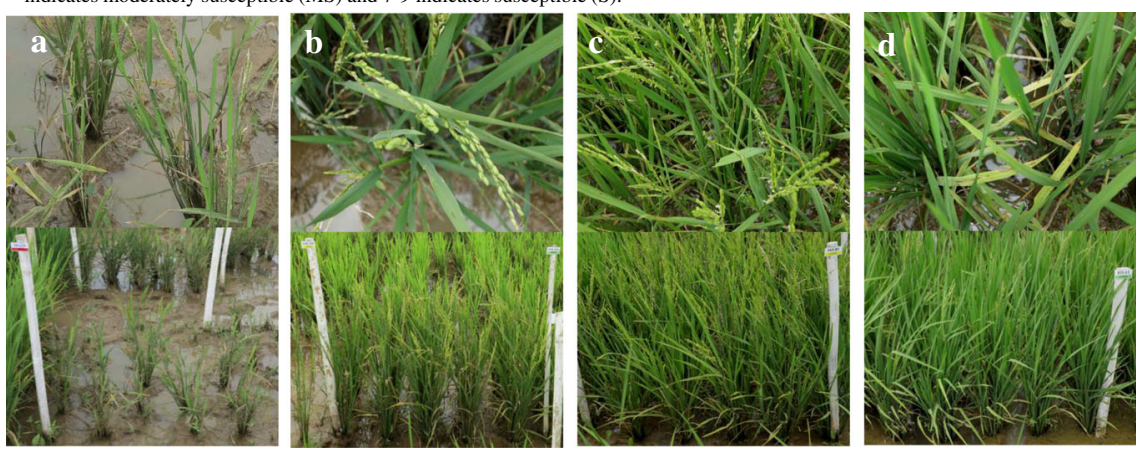

Fig. 2 Field assessment of the introgression lines IR126181 (Pi2/9-A15), IR126183 (Pi2/9-A42), IR126184 (Pi2/9-A53), IRBLz5-CA (Pi2), IRBL9-W (Pi9) and IRBLzt-T (Piz-t) together with the susceptible control CO39 and LTH. a Scoring of the introgression lines in the field with three replicates for each line. b Photograph of the disease reaction of introgression lines of $b$ IR126181, c IR126183, $d$ IR126184 and a susceptible control CO39

specific markers for known alleles, Pi2/9-RH can also be applied for the identification of novel alleles. Then, sequences of Pi2/9 homologues in Pi2/9-RH positive lines helped the clustering of identical haplotypes to avoid redundant functional characterization. Last, the generation of monogenic rice lines each containing individual novel Pi2/9 haplotypes allowed the functional validation and spectrum analysis, demonstrating an alternative approach for the functional characterization of the Pi2/9 locus containing resistant alleles. Moreover, the derived new monogenic lines can be freely distributed for field tests in different countries, providing valuable materials for resistance spectrum/frequency analysis of novel Pi2/9 alleles against rice blast under different environments. Taken together, the integrated approach presented herein provides an efficient scheme for identifying novel Pi2/9 alleles, which could also be applied to allele mining at other $R$-gene loci.
The Pi2/9 locus harbors promising alleles conferring broad-spectrum resistance against rice blast

It has been documented that the resistance spectra of different $R$ genes are mainly determined by the frequency of cognate Avr genes in the rice blast pathogen population. Therefore, the mechanisms underlying the recognition of their cognate Avr genes determine the evolution of resistance spectra controlled by different $R$-gene alleles at the same locus. In general, two main scenarios were demonstrated in the rice and rice blast phytopathosystem with respect to $R / A v r$ recognition. The first scenario captures a step-wise arms race between different $R$-gene alleles and different $A v r$-gene haplotypes. For example, the Pik locus harbors multiple $P i k$ alleles activating resistance to rice blast by recognizing different AvrPik haplotypes containing only one to three amino acid changes from one another (Yoshida et al. 2009; Kanzaki et al. 2012; Wu et al. 2014). In this scenario, new $R$-gene alleles containing

Table 5 Linkage analysis between blast resistance and the Pi2/9 haplotypes in introgression lines

\begin{tabular}{|c|c|c|c|c|c|c|c|c|c|}
\hline \multirow[t]{2}{*}{ Isolates } & \multirow{2}{*}{$\begin{array}{l}\mathrm{BC}_{3} \mathrm{~F}_{2} \\
\text { population }\end{array}$} & \multicolumn{3}{|c|}{ Number of progenies } & \multicolumn{3}{|l|}{ Chi-square test } & \multicolumn{2}{|c|}{ Number of susceptible progenies using Pi2/9-RH } \\
\hline & & R & S & Total & Expected ratio (R:S) & $x^{2}$ & $P$ & Positive & Negative \\
\hline MO15-21 & CO39/A15 & 550 & 170 & 720 & $3: 1$ & 0.741 & 0.389 & 0 & 170 \\
\hline MO15-21 & CO39/A42 & 433 & 30 & 463 & $15: 1$ & 0.042 & 0.838 & 0 & 30 \\
\hline $5167-1$ & CO39/A53 & 571 & 195 & 766 & $3: 1$ & 0.085 & 0.770 & 0 & 195 \\
\hline
\end{tabular}

$R$ resistance, $S$ susceptibility 
sequence differences from known ones are not assumed to necessarily alter the resistance spectrum, thus limiting the value for identifying novel $R$-gene alleles. On the contrary, the Pi2/9 locus consists of multiple alleles mediating resistance against rice blast by recognizing sequenceunrelated Avr genes, illustrating a contrasting scenario in the rice and rice blast phytopathosystem. For example, Piz- $t$ and Pi9 recognize sequence-unrelated AvrPiz- $t$ and AvrPi9, respectively, although $P i z-t$ and $P i 9$ are highly sequence-related to each other (Zhou et al. 2006, Qu et al. 2006; Wu et al. 2015; Li et al. 2009). This suggests more chances to identify novel alleles conferring distinct resistance spectra at Pi2/9 loci. Indeed, another two alleles (Pi2 and Pi50) were found to each control resistance against diverse sets of isolates via recognition of different $A v r$ genes other than AvrPi9 and AvrPiz-t (Su et al. 2015). More significantly, Pi2/9 alleles were reported to confer broad-spectrum resistance against rice blast in different rice-growing areas worldwide (Liu et al. 2002; Zhu et al. 2012). A recent study on large-scale germplasm screening for broad-spectrum resistance sources revealed that half of the 289 broad-spectrum blast-resistant genotypes harbored the Pi2 locus validated by an STS marker (Vasudevan et al. 2014). In this study, we identified 3 haplotypes containing 3 novel Pi2/9 homologues. The derived monogenic lines each controlled broad-spectrum resistance against diverse isolates. These data suggests that the Pi2/9 locus is likely responsible for the resistance against rice blast, which was also supported by the genetic analysis. However, the possibility that other $R$ gene loci tightly linked to the Pi2/9 locus conferred the resistance observed in these monogenic lines could not be excluded due to relatively low resolution of genetic mapping. Fine mapping or gene complementation tests of the candidate genes should be able to further clarify the function of novel Pi2/9 alleles in these 3 haplotypes.

It is worth noting that blast $R$ genes/alleles genetically identified from different donors in different geographic regions could be identical in sequence. For example, Pi25 from Guimei 2 is identical to Pid3 from Digu in protein sequence (Chen et al. 2011; Shang et al. 2009). It was also found that some $R$ genes/alleles from different donors differing by limited sequence variations conferred the same resistance spectrum by recognizing identical $A v r$ genes. In this regard, these different $R$ genes/alleles can be considered identical based on their resistance spectrum. For example, Pik1-KA from Kanto51 differs from $P i k-1$ from Kusabue by only four nucleotide sequences and Pik2-KA and Pik-2 are identical to each other. Both Pik1-KA and Pik were characterized to control identical resistance spectra by recognizing the same sets of AvrPik haplotypes (Zhai et al. 2011; Ashikawa et al. 2012). In this study, we found that Pi2/9-A42, Pi2/9-A15 and Piz- $t$ were, respectively, identified in 14,4 and 5 rice lines from different countries, demonstrating a wide distribution of the same $R$ genes/alleles in diverse germplasm. It is thus reasonable to assume that the $R$ genes/alleles in the same locus identified in different landraces described by different research programs could be identical in sequence or functionality, which raises a concern regarding a systemic nomenclature of rice blast $R$ genes before molecular characterization.

\section{Conclusion}

We identified three resistance germplasm containing novel $R$ genes at or tightly linked to the Pi2/9 locus which conferring broad-spectrum resistance against rice blast. The marker Pi2/9-RH which developed from the conserved $5^{\prime}$ portion of the $\mathrm{Pi2}$ sequence could be widely used as a diagnostic marker for the quick identification of resistance donors containing functional Pi2/9 alleles or unknown linked $R$ genes. The development of three new introgression lines containing the Pi2/9 introgression segment may play an important role in disease assessment and rice blast resistance breeding.

\section{Methods}

\section{Plant materials and $M$. oryzae isolates}

The rice $2 \mathrm{~K}$ panel consisting of 1400 rice accessions was obtained from the International Rice Genebank Collection (IRGC) of IRRI, Philippines. Fifty IRRI varieties, 30 IRBLs in the genetic background of Lijiangxintuanheigu (LTH), 2 IRBLs [IRBLz5-CA (CO) and IRBLzt-IR56 (CO)] in the genetic background of CO39, and other rice varieties including CO39, 9311, LTH, Taipei309 and Nipponbare used in this study were maintained at IRRI (Additional file 2: Table S1 and Table S2). Thirty-six M. oryzae isolates collected from four provinces (Laguna, Camarines Sur, Batangas and Bohol) of the Philippines in different years used in this study were maintained in the rice blast isolate collection at IRRI (Additional file 2: Table S3).

\section{Disease evaluation in greenhouse and field}

For greenhouse inoculation, 14-day-old rice seedlings (3-4 leaves) were sprayed with spore suspension $\left(1 \times 10^{5}\right.$ spores $/ \mathrm{mL}$ ) of individual rice blast isolates. The lesion types on the leaves were scored 7 days post-inoculation using the $0-5$ standard scale (Campbell et al. 2004). In this study, plants having lesion scores of 0,1 and 2 were considered as resistant (R), of 3 were considered as partial resistance (PR), and of scores of 4 and 5 were considered as susceptible (S). For the field evaluation, different rice lines in a $60 \mathrm{~cm} \times 30 \mathrm{~cm}$ plot were grown in a randomized complete block design with three replications at the hotspot experimental site in Ubay, Bohol, the Philippines. Disease evaluation was carried out at 40 days after transplanting when the blast disease reached to the peak. 
Disease severity was scored by following the 0-9 standard scale developed by IRRI (IRRI 2002).

\section{Screening for resistant rice germplasm accessions}

Three rounds of screening were employed to identify resistant rice germplasm in the $2 \mathrm{~K}$ panel. First, two $M$. oryzae isolates (CA89 and M64-1-3-9-1) were used to inoculate all the 1400 rice accessions in greenhouse, which led to the identification of 356 resistant accessions (Additional file 2: Table S1). Out of the 356 resistant accessions, 200 were randomly selected for the second round of screening with another two isolates (JMB8401 and M101-1-2-9-1) whereas another 156 lines were used for determining the frequency of Pi2 orthologues (Additional file 2: Table S1). Fifty-five out of 200 lines were found either resistant or partially resistant to all 4 isolates (Additional file 2: Table S1). These 55 resistant lines were further inoculated with 5 more isolates (9239-4, CA41, IK81-25, M36-1-3-10-1 and M39-1-3-8-1) for the analysis of resistance spectrum (Additional file 2: Table S1).

\section{DNA extraction and PCR amplification}

Genomic DNA of all the rice accessions was extracted from the leaf samples by using the CTAB DNA extraction method. PCR amplification using the prime pairs for Pi2/9-RH (Table 1) was carried out using the following profile: initial DNA denaturation at $95{ }^{\circ} \mathrm{C}$ for $5 \mathrm{~min}$; followed by 35 cycles of denaturation at $98{ }^{\circ} \mathrm{C}$ for $10 \mathrm{~s}$, annealing at $58{ }^{\circ} \mathrm{C}$ for $30 \mathrm{~s}$ and extension at $72{ }^{\circ} \mathrm{C}$ for $80 \mathrm{~s}$; and final extension at $72{ }^{\circ} \mathrm{C}$ for $5 \mathrm{~min}$.

\section{RT-PCR amplification and DNA sequencing}

Total RNA was isolated from the leaf tissue by using TRIZOL Reagent (Life Technologies) according to the manufacturer's instructions. In brief, $100 \mathrm{mg}$ of leave was ground using a mortar and pestle with liquid nitrogen, and the powder was suspended in $1 \mathrm{ml}$ of Trizol. Following by $10 \mathrm{~min}$ of incubation, $0.2 \mathrm{~mL}$ of chloroform was added, and samples were mixed manually for $20 \mathrm{~s}$ and then incubated for $4 \mathrm{~min}$. After centrifugation (rcf $11,000 \mathrm{~g}$ ) for $15 \mathrm{~min}$ at $4{ }^{\circ} \mathrm{C}$, the aqueous layer was retrieved and mixed with $0.25 \mathrm{~mL}$ of $3 \mathrm{M}$ sodium acetate, $\mathrm{pH} 5.2$, and $0.25 \mathrm{~mL}$ of isopropanol. A pellet was obtained by centrifugation ( $\mathrm{rcf} 11,000 \mathrm{~g}$ ) and then washed twice with $75 \%$ ethanol. After treatment by DNaseI (DNA-free TM Kit, Ambion), purified RNA was proceed with reverse transcription using the Super Script III First-strand kit (Invitrogen) to obtain first-strand cDNA. RT-PCR was further preceded by using the primer pair 5UTR-F/3UTR-R for the amplification of full-length cDNA of Pi2/9 homologues (Table 1). The 3' portions of the $P i 2 / 9$ homologues containing the sequence variations from one another were sequenced at MACROGENE Company (Korea).
Bioinformatics analysis of $\mathrm{Pi2}$ and $\mathrm{Nbs2}-\mathrm{Pi2}$ orthologue in $3 \mathrm{~K}$ genomes

The promoter sequences of $\mathrm{Pi} 2$ and Nbs2-Pi2 were aligned to the reference genome sequence (Nipponbare RGAP 7.0, http://rice.plantbiology.msu.edu) to check whether these sequences were in the reference genome or not (Additional file 1: Figure S4). After confirming that the promoter sequences of $\mathrm{Pi} 2$ and $\mathrm{Nbs}$-Pi2 were not in the reference genome, we aligned all sequencing reads from the $3 \mathrm{~K}$ panel to the reference genome to collect the unmapped sequences. The unmapped reads were used in identifying the varieties that contain the Pi2 and Nbs2$\mathrm{Pi} 2$ promoter sequences. The unmapped reads of the $3 \mathrm{~K}$ panel were aligned separately from the Pi2 and Nbs2-Pi2 promoter sequences as the reference genome using the BWA-PICARD-SAMTOOLS pipeline (Additional file 1: Figure S4). This pipeline was used to generate the alignment file (bam), and then a custom perl script was used to detect the presence of the Pi2 and Nbs2-Pi2 promoter sequences in each variety of the $3 \mathrm{~K}$ panel (Additional file 1 : Figure S4).

\section{Computational analysis}

Chi square test was conducted for the segregation of resistant and susceptible plants. The DNA sequences were edited with Sequencher (http://www.genecodes.com) and the edited sequences were aligned with Clustal Omega (http://www.ebi.ac.uk/Tools/msa/clustalo/).

\section{Additional files}

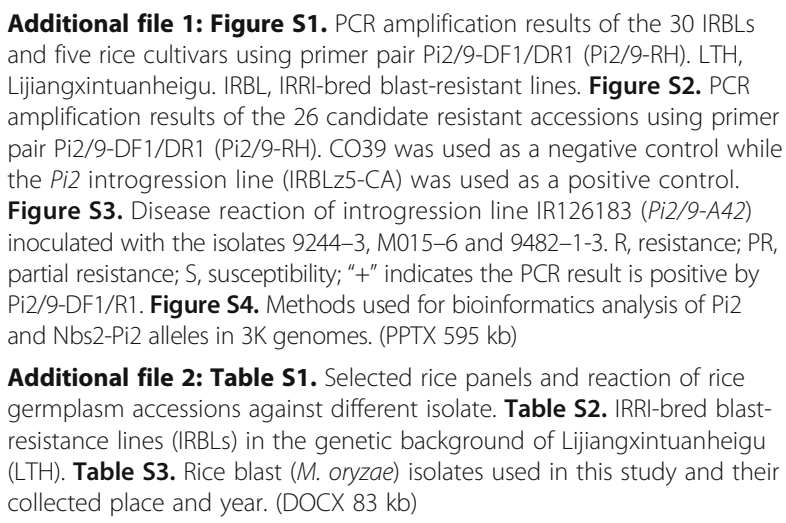

Additional file 2: Table S1. Selected rice panels and reaction of rice germplasm accessions against different isolate. Table S2. IRRI-bred blastresistance lines (IRBLs) in the genetic background of Lijiangxintuanheigu (LTH). Table S3. Rice blast (M. oryzae) isolates used in this study and their collected place and year. (DOCX $83 \mathrm{~kb}$ )

\section{Acknowledgements \\ We appreciate Mr. Bill Hardy and Dr. Hei Leung for their thorough editing of the manuscript. This work was supported, in part, by grants from the National Natural Science Foundation of China for G. L (31461143019), and the Global Rice Science Partnership (GRiSP), the SCPRID program of BBSRC and Bill \& Melinda Gates Foundation for B. Z.}

\section{Authors' contributions}

GX and BZ conceived and designed the experiments. GX, FNB, RM, JP, MJTY, $J Y, G L$ performed the experiments. GX, RM, GL and BZ analyzed the data. GX, MDS and BZ wrote the manuscript. All authors read and approved the final manuscript. 


\section{Competing interests}

The authors declare that they have no competing interests.

\section{Publisher's Note}

Springer Nature remains neutral with regard to jurisdictional claims in published maps and institutional affiliations.

\section{Author details}

'Genetics and Biotechnology Division, International Rice Research Institute, DAPO Box 7777, Metro Manila, Philippines. ${ }^{2}$ Institute of Biological Sciences, University of the Philippines Los Baños, 4031 Laguna, Philippines. ${ }^{3}$ Fujian Agriculture and Forest University, Fuzhou 350002, China.

\section{Received: 18 May 2017 Accepted: 31 July 2017}

\section{Published online: 04 August 2017}

\section{References}

Ashikawa I, Hayashi N, Yamane H, Kanamori H, Wu J, Matsumoto T, Ono K, Yano M (2008) Two adjacent nucleotide-binding site-leucine-rich repeat class genes are required to confer Pikm-specific rice blast resistance. Genetics 180:2267-2276

Ashikawa I, Hayashi N, Abe F, Wu J, Matsumoto T (2012) Characterization of the rice blast resistance gene Pik cloned from Kanto51. Mol Breeding 30:485-494

Ashkani S, Rafii M, Shabanimofrad M, Ghasemzadeh A, Ravanfar S, Latif M (2014) Molecular progress on the mapping and cloning of functional genes for blast disease in rice (Oryza sativa L.): current status and future considerations. Crit Rev Biotechnol 36:353-367

Ashkani S, Rafii M, Shabanimofrad M, Miah G, Sahebi M, Azizi P, Tanweer F, Akhtar M, Nasehi A (2015) Molecular Breeding Strategy and Challenges Towards Improvement of Blast Disease Resistance in Rice Crop. Front Plant Sci 6:886

Bhullar NK, Zhang Z, Wicker T, Keller B (2010) Wheat gene bank accessions as a source of new alleles of the powdery mildew resistance gene Pm3: a large scale allele mining project. BMC Plant Biol 10:88

Campbell MA, Chen D, Ronald PC (2004) Development of Co-Dominant Amplified Polymorphic Sequence Markers in Rice that Flank the Magnaporthe grisea Resistance Gene Pi7(t) in Recombinant Inbred Line 29. Phytopathology 94:302-307

Chen J, Shi Y, Liu W, Chai R, Fu Y, Zhuang J, Wu J (2011) A Pid3 allele from rice cultivar Gumei2 confers resistance to Magnaporthe oryzae. J Genet Genomics 38:209-216

Chen J, Peng P, Tian J, He Y, Zhang L, Liu Z, Yin D, Zhang Z (2015) Pike, a rice blast resistance allele consisting of two adjacent NBS-LRR genes, was identified as a novel allele at the Pik locus. Mol Breed 35:117

Costanzo S, Jia Y (2010) Sequence variation at the rice blast resistance gene Pi-km locus: Implications for the development of allele specific markers. Plant Sci 178:523-530

Dai L, Wu J, Li X, Wang X, Liu X, Jantasuriyarat C, Kudrna D, Yu Y, Wing AR, Han B, Zhou B, Wang G (2010) Genomic structure and evolution of the Pi2/9 locus in wild rice species. Theor Appl Genet 121:295-309

Deng Y, Zhu X, Shen Y, He Z (2006) Genetic characterization and fine mapping of the blast resistance locus Pigm(t) tightly linked to Pi2 and Pi9 in a broad-spectrum resistant Chinese variety. Theor Appl Genet 113:705-713

Fjellstrom R, McClung AM, Shank AR (2006) SSR markers closely linked to the Pi-z locus are useful for selection of blast resistance in a broad array of rice germplasm. Mol Breed 17:149-157

Fukuoka S, Yamamoto S, Mizobuchi R, Yamanouchi U, Ono K, Kitazawa N, Yasuda N, Fujita Y, Thi Thanh Nguyen T, Koizumi S, Sugimoto K, Matsumoto T, Yano M (2014) Multiple functional polymorphisms in a single disease resistance gene in rice enhance durable resistance to blast. Sci Rep 4:1-7

Hayashi K, Hashimoto N, Daigen M, Ashikawa I (2004) Development of PCR-based SNP markers for rice blast resistance genes at the Piz locus. Theor Appl Genet 108:1212-1220

Hayashi K, Yoshida H, Ashikawa I (2006) Development of PCR-based allele-specific and InDel marker sets for nine rice blast resistance genes. Theor Appl Genet 113:251-260

Hua L, Wu J, Chen C, Wu W, He X, Lin F, Wang L, Ashikawa I, Matsumoto T, Wang L, Pan Q (2012) The isolation of Pi1, an allele at the Pik locus which confers broad spectrum resistance to rice blast. Theor Appl Genet 125:1047-1055

IRRI (2002) Standard Evaluation System for Rice (SES). International Rice Research Institute, Manila, p 1-56
Jeung J, Kim B, Cho Y, Han S, Moon H, Lee Y, Jena K (2007) A novel gene, Pi40(t), linked to the DNA markers derived from NBS-LRR motifs confers broad spectrum of blast resistance in rice. Theor Appl Genet 115:1163-1177

Jiang N, Li Z, Wu J, Wang Y, Wu L, Wang S, Wen T, Liang Y, Sun P, Liu J, Dai L, Wang Z, Wang C, Luo M, Liu X, Wang G (2012) Molecular mapping of the Pi2/9 allelic gene Pi2-2 conferring broad-spectrum resistance to Magnaporthe oryzae in the rice cultivar Jefferson. Rice 5:29

Jiang J, Mou T, Yu H, Zhou F (2015) Molecular breeding of thermo-sensitive genic male sterile (TGMS) lines of rice for blast resistance using Pi2 gene. Rice 8:11

Kang H, Wang Y, Peng S, Zhang Y, Xiao Y, Wang D, Qu S, Li Z, Yan S, Wang Z, Liu W, Ning Y, Korniliev P, Leung H, Mezey J, McCouch SR, Wang G (2015) Dissection of the genetic architecture of rice resistance to the blast fungus Magnaporthe oryzae. Mol Plant Pathol 17:959-972

Kanzaki H, Yoshida K, Saitoh H, Fujisaki K, Hirabuchi A, Alaux L, Fournier E, Tharreau D, Terauchi R (2012) Arms race co-evolution of Magnaporthe oryzae AVR-Pik and rice Pik genes driven by their physical interactions. Plant J 72:894-907

Leung H, Raghavan C, Zhou B, Oliva R, Choi I, Lacorte V, Jubay M, Cruz C, Gregorio G, Singh R, Ulat V, Borja F, Mauleon R, Alexandrov NN, McNally KL, Sackville Hamilton R (2015) Allele mining and enhanced genetic recombination for rice breeding. Rice 8:34

Li W, Wang B, Wu J, Lu G, Hu Y, Zhang X, Zhang Z, Zhao Q, Feng O, Zhang H, Wang Z, Wang G, Han B, Wang Z, Zhou B (2009) The Magnaporthe oryzae avirulence gene AvrPiz-t encodes a predicted secreted protein that triggers the immunity in rice mediated by the blast resistance gene Piz-t. Mol Plant-Microbe Interact 22:411-420

Liu G, Lu G, Zeng L, Wang GL (2002) Two broad-spectrum blast resistance genes, Pig(t) and Pi2(t), are physically linked on rice chromosome 6. Mol Gen Genomics 267:472-480

Liu J, Hu Y, Ning Y, Jiang N, Wu J, Jeon J-S, Xiao Y, Liu X, Dai L, Wang G-L (2011) Genetic Variation and Evolution of the Pi9 Blast Resistance Locus in the AA Genome Oryza Species. J Plant Biol 54:294

Liu Y, Liu B, Zhu X, Yang J, Bordeos A, Wang G, Leach J, Leung H (2013) Finemapping and molecular marker development for Pi56(t), a NBS-LRR gene conferring broad-spectrum resistance to Magnaporthe oryzae in rice. Theor Appl Genet 126:985-998

Luo S, Zhang Y, Hu Q, Chen J, Li K, Lu C, Liu H, Wang W, Kuang H (2012) Dynamic Nucleotide-Binding Site and Leucine-Rich Repeat-Encoding Genes in the Grass Family. Plant Physiol 159:197-210

Lv Q, Xu X, Shang J, Jiang G, Pang Z, Zhou Z, Wang J, Liu Y, Li T, Li X, Xu J, Cheng Z, Zhao X, Li S, Zhu L (2013) Functional analysis of Pid3-A4, an ortholog of rice blast resistance gene Pid3 revealed by allele mining in common wild rice. Phytopathology 103:594-599

Ma J, Lei C, Xu X, Hao K, Wang J, Cheng Z, Ma X, Ma J, Zhou K, Zhang X, Guo X, Wu F, Lin Q, Wang C, Zhai H, Wang H, Wan J (2015) Pi64, encoding a novel CC-NBS-LRR Protein, confers resistance to leaf and neck blast in rice. Mol Plant-Microbe Interact 28:558-568

Okuyama Y, Kanzaki H, Abe A, Yoshida K, Tamiru M, Saitoh H, Fujibe T, Matsumura H, Shenton M, Galam D, Undan J, Ito A, Sone T, Terauchi R (2011) A multifaceted genomics approach allows the isolation of the rice Pia-blast resistance gene consisting of two adjacent NBS-LRR protein genes. Plant J 66:467-479

Qu S, Liu G, Zhou B, Bellizzi M, Zeng L, Dai L, Han B, Wang G (2006) The broad-spectrum blast resistance gene Pi9 encodes a nucleotide-binding site-leucine-rich repeat protein and is a member of a multigene family in rice. Genetics 172:1901-1914

Ramkumar G, Srinivasarao K, Mohan K, Sudarshan I, Sivaranjani AKP, Gopalakrishna K, Neeraja CN, Balachandran SM, Sundaram RM, Prasad MS, Shobha Rani N, Rama Prasad AM, Viraktamath BC, Madhav MS (2011) Development and validation of functional marker targeting an InDel in the major rice blast disease resistance gene Pi54 $\left(\right.$ Pik $\left.^{h}\right)$. Mol Breed 27:129-135

Shang J, Tao Y, Chen X, Zou Y, Lei C, Wang J, Li X, Zhao X, Zhang M, Lu Z, Xu J, Cheng $Z$, Wan J, Zhu L (2009) Identification of a new rice blast resistance gene, Pid3, by genomewide comparison of paired nucleotide-binding siteleucine-rich repeat genes and their pseudogene alleles between the two sequenced rice genomes. Genetics 182:1303-1311

Skamnioti P, Gurr SJ (2009) Against the grain: safeguarding rice from rice blast disease. Trends Biotechnol 27:141-150

Su J, Wang W, Han J, Chen S, Wang C, Zeng L, Feng A, Yang J, Zhou B, Zhu X (2015) Functional divergence of duplicated genes results in a novel blast resistance gene Pi50 at the Pi2/9 locus. Theor Appl Genet 128:2213-2225

Takagi H, Uemura A, Yaegashi H, Tamiru M, Abe A, Mitsuoka C, Utsushi H, Natsume S, Kanzaki H, Matsumura H, Saitoh H, Yoshida K, Cano LM, Kamoun 
S, Terauchi R (2013) MutMap-Gap: Whole-genome resequencing of mutant $\mathrm{F}_{2}$ progeny bulk combined with de novo assembly of gap regions identifies the rice blast resistance gene Pii. New Phytol 200:276-283

Takahashi A, Hayashi N, Miyao A, Hirochika H (2010) Unique features of the rice blast resistance Pish locus revealed by large scale retrotransposon-tagging. BMC Plant Biol 10:175

Tanweer FA, Rafii MY, Sijam K, Rahim HA, Ahmed F, Latif MA (2015) Current advance methods for the identification of blast resistance genes in rice. $C R$ Biol 338:321-334

Thakur S, Singh PK, Rathour R, Variar M, Prashanthi SK, Gopalakrishnan S, Singh AK, Singh UD, Chand D, Singh NK, Sharma TR (2014) Genotyping and development of single-nucleotide polymorphism (SNP) markers associated with blast resistance genes in rice using GoldenGate assay. Mol Breed 3: 1449-1463

Tian D, Chen Z, Chen Z, Zhou Y, Wang Z, Wang F, Chen S (2016) Allele-specific marker-based assessment revealed that the rice blast resistance genes $\mathrm{Pi2}$ and $P i 9$ have not been widely deployed in Chinese indica rice cultivars. Rice 9:19

Valent B, Khang CH (2010) Recent advances in rice blast effector research. Curr Opin Plant Biol 13:434-441

Vasudevan K, Vera Cruz CM, Gruissem W, Bhullar NK (2014) Large scale germplasm screening for identification of novel rice blast resistance sources. Front Plant Sci 5:505

Vasudevan K, Gruissem W, Bhullar NK (2015) Identification of novel alleles of the rice blast resistance gene Pi54. Sci Rep 5:1-11

Wang G, Mackill D, Bonman J, McCouch S, Champoux M, Nelson R (1994) RFLP mapping of genes conferring complete and partial resistance to blast in a durably resistant rice cultivar. Genetics 136:1421-1434

Wang Y, Wang D, Deng X, Liu J, Sun P, Liu Y, Huang H, Jiang N, Kang H, Ning Y, Wang Z, Xiao Y, Liu X, Liu E, Dai L, Wang G (2012) Molecular mapping of the blast resistance genes Pi2-1 and Pi51(t) in the durably resistant rice "Tianjingyeshengdao". Phytopathology 102:779-786

Wu W, Wang L, Zhang S, Li Z, Zhuang Y, Lin F, Pan Q (2014) Stepwise arms race between AvrPik and Pik alleles in the rice blast pathosystem. Mol PlantMicrobe Interact 27:759-769

Wu J, Kou Y, Bao J, Li Y, Tang M, Zhu X, Ponaya A, Xiao G, Li J, Li C, Song M, Cumagun C, Deng Q, Lu G, Jeon J, Naqvi NI, Zhou B (2015) Comparative genomics identifies the Magnaporthe oryzae avirulence effector AvrPi9 that triggers Pi9-mediated blast resistance in rice. New Phytol 206:1463-1475

Yoshida K, Saitoh H, Fujisawa S, Kanzaki H, Matsumura H, Yoshida K, Tosa Y, Chuma I, Takano Y, Win J, Kamoun S, Terauchi R (2009) Association genetics reveals three novel avirulence genes from the rice blast fungal pathogen Magnaporthe oryzae. Plant Cell 21:1573-1591

Yuan B, Zhai C, Wang W, Zeng X, Xu X, Hu H, Lin F, Wang L, Pan (2011) The Pik-p resistance to Magnaporthe oryzae in rice is mediated by a pair of closely linked CC-NBS-LRR genes. Theor Appl Genet 122:1017-1028

Zhai C, Lin F, Dong Z, He X, Yuan B, Zeng X, Wang L, Pan Q (2011) The isolation and characterization of Pik, a rice blast resistance gene which emerged after rice domestication. New Phytol 189:321-334

Zhai C, Zhang Y, Yao N, Lin F, Liu Z, Dong Z, Wang L, Pan Q (2014) Function and interaction of the coupled genes responsible for Pik-h encoded rice blast resistance. PLoS One 9(6):e98067

Zhou B, Qu S, Liu G, Dolan M, Sakai H, Lu G, Bellizzi M, Wang G (2006) The eight amino-acid differences within three leucine-rich repeats between Pi2 and Piz- $t$ resistance proteins determine the resistance specificity to Magnaporthe grisea. Mol Plant-Microbe Interact 19:1216-1228

Zhou B, Dolan M, Sakai H, Wang G (2007) The genomic dynamics and evolutionary mechanism of the Pi2/9 locus in rice. Mol Plant-Microbe Interact 20:63-71

Zhu X, Chen S, Yang J et al (2012) The identification of Pi5O(t), a new member of the rice blast resistance Pi2/Pi9 multigene family. Theor Appl Genet 124:1295-1304

\section{Submit your manuscript to a SpringerOpen ${ }^{\circ}$ journal and benefit from:}

- Convenient online submission

- Rigorous peer review

- Open access: articles freely available online

- High visibility within the field

- Retaining the copyright to your article

Submit your next manuscript at $\boldsymbol{\nabla}$ springeropen.com 